\title{
Umeå University
}

This is an accepted version of a paper published in Polymer. This paper has been peer-reviewed but does not include the final publisher proof-corrections or journal pagination.

Citation for the published paper:

Yu, J., Gröbner, G., Tonpheng, B., Andersson, O. (2011)

"Microstructure, nucleation and thermal properties of high-pressure crystallized MWCNT/nylon-6 composites"

Polymer, 52(24): 5521-5527

URL: http://dx.doi.org/10.1016/j.polymer.2011.09.036

Access to the published version may require subscription.

Permanent link to this version:

http://urn.kb.se/resolve?urn=urn:nbn:se:umu:diva-49310

http://umu.diva-portal.org 


\title{
Microstructure, nucleation and thermal properties of high-pressure crystallized MWCNT/nylon-6 composites
}

\author{
Junchun $\mathrm{Yu}^{1}$, Gerhard Gröbner ${ }^{2}$, Bounphanh Tonpheng ${ }^{1}$, and Ove Andersson ${ }^{1 *}$ \\ ${ }^{1}$ Department of Physics, Umeå University, 90187 Umeå, Sweden \\ ${ }^{2}$ Department of Chemistry, Umeå University, 90187 Umeå, Sweden
}

* Corresponding author. Tel: +46 90 7865034; Fax: +46 907866673.

E-mail address: ove.andersson@physics.umu.se (O. Andersson) 


\section{ABSTRACT}

Multi-wall carbon nanotube (MWCNT)/nylon-6 composites made by in-situ polymerization and subsequently modified by treatment at $1.0 \mathrm{GPa}$ (or $1.7 \mathrm{GPa}$ ) and $530 \mathrm{~K}$ have been studied by WAXD, DSC and NMR. The pressure treatment gives an amorphous to crystalline transformation where the crystallinity increases from $\sim 31 \%$ to as much as $\sim 58 \%$ concurrently as the nylon-6 crystals increase in size and attain a preferred orientation relative to the applied pressure. A composite of $2.1 \mathrm{wt} \%$ purified MWCNT in nylon-6 shows significantly higher melting temperature than neat nylon-6 after identical pressure treatments. The improved thermal stability of the composite is attributed to crystal growth in the presence of reinforcing MWCNTs. The NMR spectrum of a pressure treated composite is similar to that of nylon-6 single crystals, which suggests a reduction of crystal boundaries after treatment, but there is no indication of covalent bonds between the nylon-6 chains and the MWCNTs.

Keywords: Multi-wall carbon nanotube, nylon-6, thermal property, crystallization, nucleation 


\section{Introduction}

High-pressure studies of polymers and polymer based composite materials provide interesting possibilities to gain understanding of complex materials and to make new materials, e.g. by changing the structure and mobility via pressure induced phase and/or glass transitions. This is especially attractive when strong densification induces irreversible transformations as it opens up the possibility of recovering samples for detailed analysis of microstructural changes. These can then be linked to changes in properties, which may be uniquely modified by a treatment under simultaneous high-pressure-high-temperature (HP\&HT) conditions. As an example, the fullerene $\mathrm{C}_{60}$ shows both glass and phase transitions under pressure [1]. It can be irreversibly cross-linked into various polymeric structures [2] and it has been reported to form super-hard carbon structures under pressure [3]. In the cases of polymers and their composites, HP\&HT treatments are particularly useful for irreversibly changing two of the most important polymer characteristics: cross-link density and degree of crystallinity. HP\&HT treatments can initiate cross-link processes in, e.g., polybutadiene $[4,5]$ and polyisoprene [6] and produce a densified network polymer without the use of cross-linking agents. Moreover, the crystallinity can be significantly improved, which has been demonstrated for a number of polymers such as polyethylene [7] and nylon, e.g. that of nylon- 6 can be increased from $\sim 30 \%$ to $~ 60 \%$ [8]. In the context of composites, these features can thus be exploited to investigate the possibility of augmented effects of fillers on the properties [9].

Carbon nanotube (CNT)/nylon-6 composites have recently been synthesized and studied extensively to establish the effect of CNT fillers and to achieve nylon materials with better electrical conductance and improved thermal and mechanical properties [10-17]. As for other CNT/polymer composites, it has been shown that multi-wall carbon nanotube (MWCNT) fillers significantly improve the electrical conductivity [12]. Moreover, Gao et al. [16, 17] have studied the mechanical reinforcement after grafting nylon-6 chains on - $\mathrm{COOH}$ functionalized single-wall carbon nanotubes (SWCNTs) and reported improved Young's modulus, tensile strength, and thermal stability of the SWCNT-reinforced nylon-6. Jose et al. [13] studied MWCNT/nylon-6 composites produced by electrospinning and reported a preferred orientation of the nylon-6 chains and the MWCNTs along the flow direction, which was envisaged by electron microscopy. They also used differential scanning calorimetry (DSC) to study the effect of MWCNTs on the 
recrystallization behavior of nylon-6 [13] and found higher recrystallization temperature than for pure nylon-6 [15], in agreement with corresponding results of nylon-12 [18].

Here we report the microstructural and thermal stability changes after a sluggish coldcrystallization transition in MWCNT/nylon-6 composites, which occurs under HP\&HT treatment. The transition was previously established through high-pressure, in-situ, thermal conductivity measurements, which showed that the thermal conductivity of the highly crystallized state increased $~ 37 \%$ [19]. In this study, we have characterized the HP\&HT treated samples of: nylon6, purified MWCNT filled nylon-6 (P-composite) and functionalized MWCNT filled nylon-6 (Fcomposite), and the corresponding untreated (virgin) samples by WAXD, DSC and solid state NMR spectroscopy. The purpose was to determine the structural changes, their effect on the thermal stability and transition behavior, and the role of CNTs in these structural and property changes.

\section{Experimental section}

\subsection{Materials and pre-treatment}

MWCNTs produced by catalytic chemical vapor deposition were purchased from Nanocyl (3150 and 3151). Transmission electron microscopy analyses showed that Nanocyl 3150 and 3151 had average lengths of $\sim 1 \mu \mathrm{m}$ and $\sim 0.5 \mu \mathrm{m}$, respectively. Nanocyl 3151 (F-MWCNTs) with less than $4 \%$ of $-\mathrm{COOH}$ functionalized MWCNTS, which had a stated carbon purity of $>95 \%$ and a metal oxide content of $<5 \%$, was used without further purification. Nanocyl 3150 (PMWCNTs) with a carbon purity of $>95 \%$ and a metal oxide content of $<5 \%$ was further purified before use. A typical purification procedure included oxidation in air, washing in $37 \% \mathrm{HCl}$ acid (Sigma-Aldrich, ACS reagent) and, subsequently, rinsing by de-ionized and purified water (Milli$\mathrm{Q}^{\circledR}$ Ultrapure WaterSystem), filtration and drying [20]. The synthesis of $2.1 \mathrm{wt} \%$ MWCNT/nylon-6 composite and neat nylon-6 followed the steps suggested by Gao et al. [16, 17] and has been previously described in detail [19]. The as-made nylon-6 had a viscosity-average molecular weight of 28000 as determined by a falling-ball viscometer (Thermo Scientific HAAKE falling ball viscometer type B). The 2.1 wt.\% F-MWCNTs/nylon-6 composite was prepared for promoting the possibility of covalent bonding between the filler and the matrix at HP\&HT conditions. (-COOH groups of the functionalized MWCNTs may react with $-\mathrm{NH}_{2}$ in nylon-6.). The higher treatment pressure (1.7 GPa) compared to that selected for the PMWCNTs/nylon-6 composite (1.0 GPa) was chosen both to promote reactivity of the 
functionalized CNTs and to test another pressure for the possibility of cross-linking of nylon-6. (Previous tests were done at 1.0 and 1.2 GPa [8].) But the subsequent analysis of the HP\&HT treated sample showed no signs of covalent bonds between F-MWCNT and nylon-6 or crosslinks in the nylon-6 matrix.

\subsection{HP\&HT experimental setup}

The MWCNT/nylon-6 and nylon-6 samples were hot pressed into plates (diameter: 37 mm, thickness: $\sim 3 \mathrm{~mm}$ ) at $250^{\circ} \mathrm{C}$ in an argon atmosphere and thereafter degassed in an vacuum oven at $80{ }^{\circ} \mathrm{C}$ for $24 \mathrm{~h}$ before inserted in a custom made Teflon cell. The cell fitted in a piston-cylinder device, as described in detail previously [8]. The pressure was generated by a hydraulic press with inaccuracy of $\pm 40 \mathrm{MPa}$ at $1.0 \mathrm{GPa}$ and the temperature was measured with an internal Chromel-Alumel thermocouple with an inaccuracy of about $\pm 0.2 \mathrm{~K}$. As established previously by thermal conductivity measurements [8, 19], nylon-6 shows a transition on heating to above $500 \mathrm{~K}$ at 1.0 GPa. The samples studied here are identical to those studied previously by thermal conductivity and heat capacity, in-situ, measurements after treatment at $1.0 \mathrm{GPa}$ (or $1.7 \mathrm{GPa}$ ) and $530 \mathrm{~K}$ for $4 \mathrm{~h}$ [19].

\subsection{Characterizations of crystallinity}

\subsubsection{Differential scanning calorimetry, DSC}

A Pyris Diamond DSC equipped with intra-cooler was used for DSC measurements. All the samples used for the DSC measurement were pumped $24 \mathrm{~h}$ in a vacuum oven to remove water and thereafter encapsulated under nitrogen or argon atmosphere at room temperature to avoid oxygen. The samples were heated from 20 to $250^{\circ} \mathrm{C}$ at a scan rate of $10^{\circ} \mathrm{C}$ per minute and held at $250{ }^{\circ} \mathrm{C}$ for 5 minutes to melt the samples before cooling. Subsequently, the samples were cooled to $20{ }^{\circ} \mathrm{C}$ using the same scan rate. The degree of crystallinity $C_{\mathrm{DSC}}$ (mass fraction) was calculated from heat of fusion using the total enthalpy method after subtracting the weight of MWCNTs [8].

\subsubsection{Wide angle $X$-ray diffraction, WAXD}

WAXD was carried out using a Siemens/Bruker D5000 diffractometer with $\mathrm{CuK} \alpha$ radiation at an acceleration voltage of $40 \mathrm{kV}$ and a tube current of $30 \mathrm{~mA}$. The samples were scanned twice from $5^{\circ}$ to $50^{\circ}(2 \theta)$ at a scanning rate of $4^{\circ}$ per minute. The first scan was done on the as recovered sample and scanned with the axis of rotation in the same direction as the applied pressure, and the second after the sample had been manually chopped into small grains. The 
WAXD spectrum of the first scan was used to determine the preferred pressure induced crystal orientation and that of the latter was used to determine the degree of crystallinity (mass fraction), $C_{W A X D}$, and crystallite size and perfection (CSP) [8].

\subsubsection{Density measurements}

The samples were weighted and the volumes were obtained by quickly immersing the samples into water at $20{ }^{\circ} \mathrm{C}$ [21]. The degree of crystallinity (mass fraction), $C_{\rho}$, was determined from the equation:[22]

$$
C_{\rho}=\left(\frac{1}{\rho_{\text {amorph }}}-\frac{1}{\rho}\right) /\left(\frac{1}{\rho_{\text {amorph }}}-\frac{1}{\rho_{\text {cryst }}}\right) \cdot 100 \%
$$

where $\rho$ is the measured density of nylon-6, $\rho_{\text {amorph }}$ is the density of the amorphous phase (1.098 $\left.\mathrm{g} \mathrm{cm}^{-3}\right)$ and $\rho_{\text {cryst }}$ is the density of crystalline nylon-6 $\left(1.220 \mathrm{~g} \mathrm{~cm}^{-3}\right)$ [23, 24]. (The density of the $2.1 \mathrm{wt} \%$ MWCNT filler was accounted for in the calculation of the nylon- 6 density of the composites.)

\subsection{Solid state nuclear magnetic resonance (NMR) spectroscopy}

Measurements were carried out on a $400 \mathrm{MHz}$ Infinity spectrometer (Chemagnetics, USA) using a $4 \mathrm{~mm}$ double resonance cross polarization magic angle spinning (CP MAS) probe. Solid state ${ }^{13} \mathrm{C}$ CP MAS NMR experiments under proton decoupling were acquired at room temperature by using a contact time of 5 ms duration and a repetition delay of $3 \mathrm{~s}$ at a MAS speed of $6 \mathrm{kHz}$. All ${ }^{13} \mathrm{C}$ NMR spectra were assigned using adamantane as an external reference.

\subsection{Imaging of samples by microscope}

Tapping mode Atomic Force Microscopy (AFM) imaging of the composites was carried out using a MultiMode AFM (Nanoscope IV Controller, Veeco Metrology) equipped with a LTESP probe (Veeco probe). For AFM studies, a semi-thin sample $(500 \mathrm{~nm})$ was cut by a Leica Reichert ultracuts ultramicrotome equipped diamond knife and subsequently placed on a silicon wafer.

Transmission electron microscopy (TEM, JEM-1230(Jeol)) equipped with a camera (Gatan MSC $600 \mathrm{CW}$ ) was used for imaging ultra-thin sections of the samples at $80 \mathrm{kV}$ (thickness $\sim 80$ $\mathrm{nm}$, which was cut by the same ultramicrotome using a diamond knife). 


\section{Results and discussion}
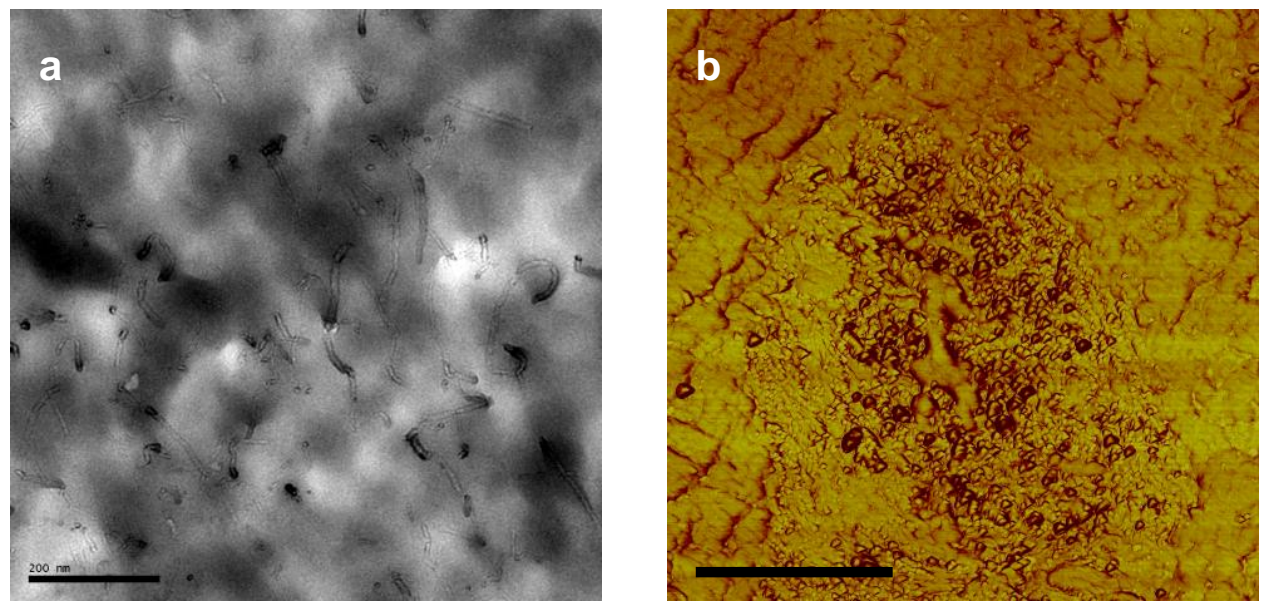

Fig. 1. (a) TEM and (b) AFM images of the P-composite after $1.0 \mathrm{GPa}$ treatment. The scale bars are $200 \mathrm{~nm}$ and $1 \mu \mathrm{m}$ in (a) and (b), respectively.

The MWCNT/nylon-6 composites synthesized by in-situ polymerization and a similarly produced neat nylon-6 sample were all subjected to high-pressure-high-temperature (HP\&HT) treatments to investigate mictrostructural changes and the effect of the MWCNT filler. The Pcomposite and nylon-6 were treated at $1.0 \mathrm{GPa}$ whereas the F-composite was treated at $1.7 \mathrm{GPa}$, (see experimental section for the difference in treatment pressure). As shown in Fig. 1a, the MWCNTs in the P-composite were well dispersed and uniformly distributed. The circular small tips in Fig. 1b are the tips (or caps) of clusters of MWCNTs in the polymer matrix.

\subsection{Structural properties and crystal orientation by WAXD}

Nylon-6 can exist in two crystalline modifications at ambient conditions: a stable monoclinic $\alpha$ structure and a monoclinic or pseudohexagonal $\gamma$ crystal structure [25]. Spectra of the former show two principal diffraction peaks: $\alpha 1$ at $19.9^{\circ}$ from (200) planes and $\alpha 2$ at $23.75^{\circ}$ due to the (002)+(202) planes [26]. The (200) are planes along the molecular chains but cutting through hydrogen-bonded sheets, whereas the (002) planes lie parallel to the hydrogen-bonded sheets with (202) as the diagonal planes [25]. The $\gamma$ structure has a principal diffraction peak at $22^{\circ}$ [25]. Amorphous nylon-6 gives a broad diffuse halo with an intensity maximum at ca. $22^{\circ}$ [25], and a sample of pure MWCNTs shows a peak at $26^{\circ}$ due to graphitized carbon [27]. 

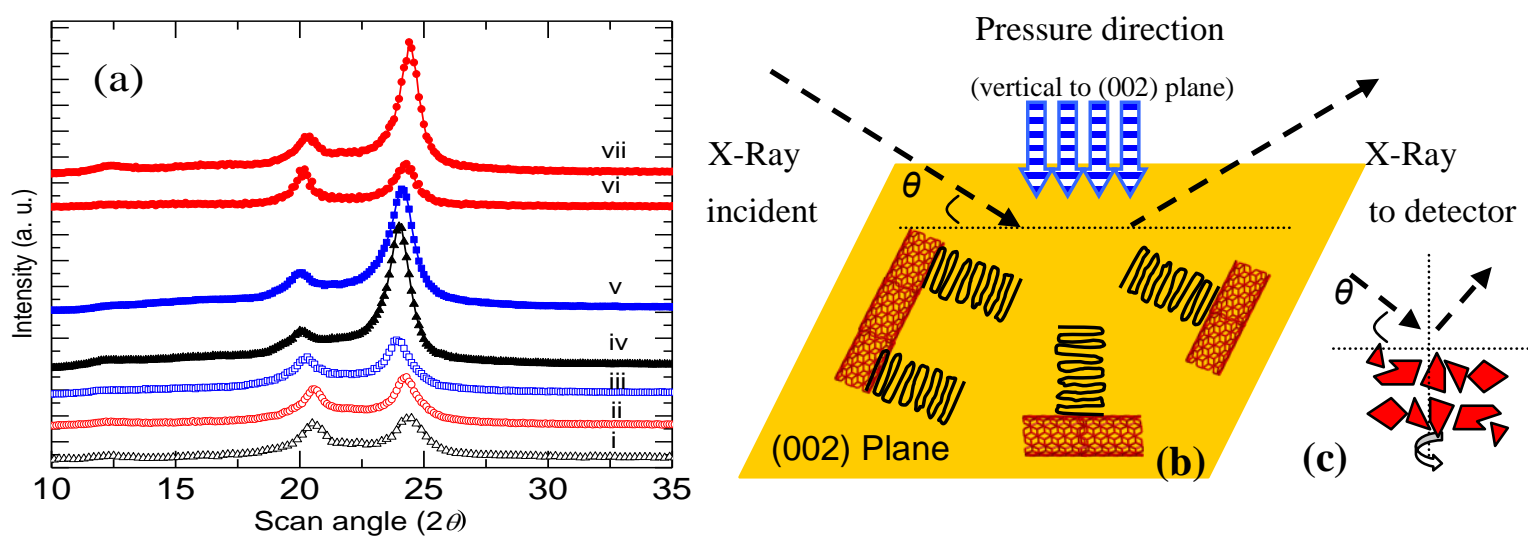

Fig. 2. (a) WAXD spectra of nylon-6 and its MWCNT composites (from bottom to top): i. nylon6; ii. P-composite; iii. F-composite; iv. Plate-shaped 1.0 GPa treated nylon-6; v. Plate-shaped 1.7 GPa treated F-composite vi. Randomly arranged particles of 1.0 GPa treated P-composite, as shown in (c); vii. Plate-shaped 1.0 GPa treated P-composite. Sample iv, v and vii were scanned with the axis of rotation of the recovered sample plates in the same direction as the pressure was applied, as shown in (b) where the sample plate surface is parallel with the (002) plane. The intensity of the spectra were normalized at the (200) reflection (near $20^{\circ}$ ) and shifted vertically for clarity. (b) Preferred growth direction of nylon-6 crystals under pressure as deduced from the WAXD spectra.

As shown in Fig. 2a, the spectra of the untreated P- and F-composites are the same as that of nylon-6, which corresponds to the $\alpha$ crystal structure with almost equal intensities for the $\alpha 1$ and $\alpha 2$ peaks. This is in agreement with Liu et al.'s [28] study of the crystal structure of nylon-6 with and without MWCNT fillers. They reported that neat nylon-6 crystallized in mainly the stable $\alpha$ structure at $200{ }^{\circ} \mathrm{C}$, and that progressively lower crystallization temperatures gave a phase mixture with increasing amount of the $\gamma$ phase. Crystallization of nylon- 6 in the presence of 1 wt $\%$ MWCNTs resulted in the $\alpha$-phase independent of crystallization temperature in the 80-200 ${ }^{\circ} \mathrm{C}$ range, which suggests that MWCNTs promote nucleation and growth of the more stable $\alpha$ phase. They noted that this is different from the behavior of nanoclay fillers, which promote the normally less stable $\gamma$ phase. For instance, montmorillonite (MMT) in nylon-6 crystallizes into the $\gamma$ phase between 185 and $205{ }^{\circ} \mathrm{C}$ [29], and for micro-extruded samples of neat nylon-6 and 2 wt\% MMT in nylon-6, the former exhibits predominantly the $\alpha$-phase whereas the latter exhibits both the $\gamma$ and $\alpha$ phases [30]. 
The spectra of the HP\&HT treated composites, shown in Fig. 2a, are the same as that of the $\alpha$ crystal structure but with significantly stronger $\alpha 2$ than $\alpha 1$. The spectra were recorded with the samples' axes of rotation in the same direction as the applied pressure (Fig.2b). The relative increase of the $\alpha_{2}$ peak suggests that the crystals attained a preferred orientation. This is also substantiated by the recovery of the original WAXD spectra for a sample chopped into small pieces, which ensures a random orientation of the crystals (Fig. 2c). The results are similar as those for HP\&HT treated commercial nylon-6 for which the treatment promotes folding within the plane perpendicular to the applied load during cold crystallization under pressure [8]. This causes the polymer chains, or more specifically the (002) planes, to become preferably oriented parallel with the sample plate surface as shown schematically in Fig 2b. But since the relative increase of the $\alpha 2$ peak is less for the HP\&HT treated P- and F-composites (Fig. 2a) than for nylon-6, it suggests that the MWCNTs weakly obstruct the tendency for the crystals to become oriented. (The intensity ratios of $\alpha 2 / \alpha 1$ in Fig 2 are: 4.8, 4.2 and 3.9 for treated nylon-6, Pcomposite and F-composite, respectively.)

The WAXD results were used to calculate the degree of crystallinity $C_{\mathrm{WAXD}}$ and crystals size and perfection (CSP) before and after the treatments. The degree of crystallinity $\left(C_{\rho}\right)$ before and after the treatments was also estimated from the densities. As shown in Table $1, C_{\mathrm{WAXD}}$ and $C_{\rho}$ do not vary much between the untreated samples. We note that the CSP is slightly larger in the untreated composites, which indicates a heterogeneous nucleation effect of the MWCNTs that makes the crystals grow at higher temperatures. Normally, this produces larger and better crystals and may also give a higher degree of crystallinity, which are in agreement with the WAXD results. Another possibility for the difference in CSP is slight molecular weight differences caused by variations in the polymerization process, e.g. temperature fluctuations. A decrease in molecular weight generally promotes the crystallinity and increases the $C S P$. In any case, the CSP of nylon-6 is not strongly affected by the MWCNTs and we also note that a decrease of CSP of nylon 6,6 was observed for samples with 50-100 $\mu \mathrm{m}$ long carboxylic acid functionalized MWCNT fillers [31].

The HP\&HT treatment caused $C_{\mathrm{WAXD}}$ and $C S P$ to increase for all samples. The increased degree of crystallinity, as indicated by $C_{\mathrm{WAXD}}$, is corroborated by the density measurements but the absolute values and changes differ. The density measurements indicate a larger increase for the composites than neat nylon-6, whereas $C_{\text {WAXD }}$ shows the reverse. The values for $C S P$ are 
about the same after the treatment, which suggest a slightly more pronounced CSP increase in neat nylon-6 than in the composites.

Table 1: Crystallinity $\left(C_{W A X D}, C_{D S C}\right.$ and $\left.C_{\rho}\right)$, density, and crystal size and perfection $(C S P)$ of virgin and treated samples.

\begin{tabular}{lcccccc}
\hline Sample & $C_{W A X D}$ & $C_{D S C}$ & $\begin{array}{l}\text { Density } \\
\left(\mathrm{g} / \mathrm{cm}^{3}\right)\end{array}$ & $C_{\rho}$ & \multicolumn{2}{c}{$C S P$} \\
& & & & & $\alpha 1(\AA)$ & $\alpha 2(\AA)$ \\
\hline Virgin nylon-6 & $39.0 \%$ & $21.0 \%$ & 1.162 & $55.1 \%$ & 74.7 & 67.7 \\
1.0 GPa treated nylon-6 & $56.0 \%$ & $56.8 \%$ & 1.182 & $71.1 \%$ & 98.3 & 83.7 \\
Virgin P-composite & $42.1 \%$ & $19.7 \%$ & 1.172 & $55.1 \%$ & 81.4 & 71.2 \\
1.0 GPa treated P-composite & $49.8 \%$ & $65.5 \%$ & 1.220 & $93.1 \%$ & 96.0 & 90.3 \\
Virgin F-composite & $41.5 \%$ & $28.5 \%$ & $1.172^{\mathrm{a}}$ & $55.1 \%$ & 90.6 & 64.9 \\
1.7 GPa treated F-composite & $48.4 \%$ & $43.9 \%$ & 1.218 & $91.6 \%$ & 100.8 & 82.0
\end{tabular}

${ }^{\mathrm{a}}$ value calculated by the mixture rule using the densities of 1.162 and $1.9 \mathrm{~g} \mathrm{~cm}^{-3}$ for nylon- 6 and MWCNTs, respectively.

\subsection{Polymorphism and cross-linking properties by solid state NMR}

Typical solid state ${ }^{13} \mathrm{C}$ CP MAS NMR spectra of the F-composite and the 1.7 GPa treated Fcomposite are shown in Fig. 3. The NMR resonances, as visible here, refer to the polymer part of the composite and are assigned according to assignment by Kubo et al. [32]. Nylon-6 has 6 magnetically nonequivalent carbons in the constitutional repeating unit, i.e. 5 methylene carbons (C1 to C5, where C3 and C4 combine into a single peak) and 1 carbonyl carbon (C6), as shown in Fig. 3. The NMR spectrum depends slightly on the phase structure, as described previously by Hatfield et al. [33], who also applied ${ }^{13} \mathrm{C}$ CP-MAS NMR. They recorded the NMR spectra for samples of highly amorphous, "high in $\alpha$ crystallinity" and "high in $\gamma$ crystallinity" nylon-6, respectively, where the two latter contained also substantial amorphous fractions but no detectable amounts of the other crystalline phase. A "high in $\alpha$ crystallinity" sample showed NMR peaks with chemical shift values at 36.7, 26.530 .443 .6 and 173.4 ppm whereas a "high in $\gamma$ crystallinity" sample showed peaks at 37.8, 30.1, 34.1, 39.9 and $173.0 \mathrm{ppm}$, which were assigned to $\mathrm{C} 1$ to C6, as shown in Fig. 3 [33]. 
The spectrum of the untreated F-composite (Fig. 3), shows the C1 to C6 peaks at 36.4, 26.4, 30.1, 43.3 and $173.5 \mathrm{ppm}$, respectively, which confirms that the F-composite possesses a $\alpha$ dominated crystalline structure. In addition to the typical C1 to C6 peaks of the nylon-6 spectrum, there appears also a low intensity spectral shoulder at $41.0 \mathrm{ppm}$, which is near that of the C5 carbon peak. This feature appears to be similar to the one at ca. 40 ppm reported by Kubo et al. [32, 34] for a highly amorphous nylon-6 sample, which was obtained by melt-quenching. Thus, the NMR results also suggest that the untreated F-composite has a crystalline fraction of mainly the $\alpha$ phase in coexistence with a significant amount of an amorphous nylon- 6 fraction.

After HP\&HT treatment, this low intensity shoulder due to amorphous nylon-6 was not visible anymore and the other NMR line widths decreased giving a spectrum strikingly similar to that of a "nylon-6 single crystal sample" reported by Kubo et al. [34] or to that of a fully crystalline form of nylon-6 as deduced by VanderHart et al. [35]. This suggests that the new $\alpha$ crystals grew in a preferred direction forming similarly oriented large crystals which gives a quasi-single crystal NMR spectrum. The enlargement and rearrangement of the nylon- 6 crystals are consistent with the WAXD results.

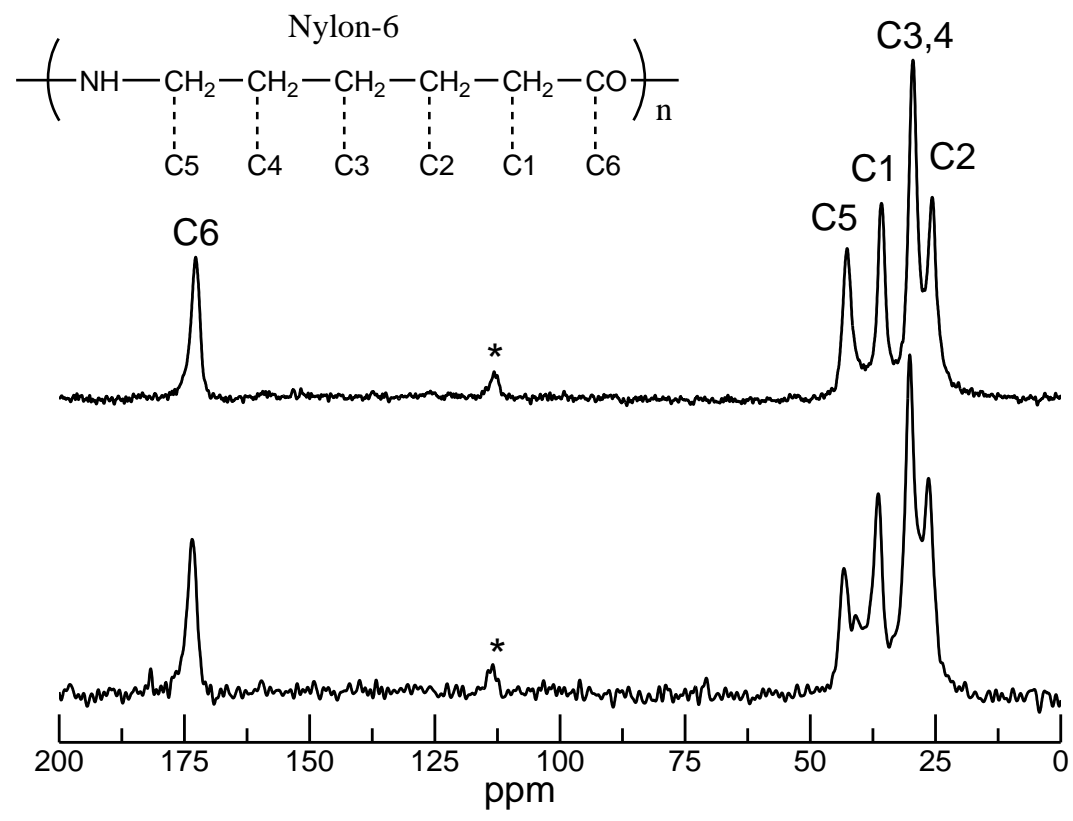

Fig. 3. Solid state ${ }^{13} \mathrm{C}$ CP MAS NMR spectra of $6 \mathrm{KHz}$ of F-composite (bottom) and F-composite treated at $1.7 \mathrm{GPa}$ (top) obtained at room temperature and a sample spinning rate of $6 \mathrm{kHz}$. Asterisks indicate spinning side bands. 
Besides changing the microstructure, it is also possible that HP\&HT treatment changes the chemical structure, e.g. by cross-linking as reported by Yang et al. [36, 37] for nylon-1010 after treatment at $523 \mathrm{~K}$ in the 1.0 to $1.2 \mathrm{GPa}$ range. Since the line width of an NMR resonance reflects dynamical processes, such as the exchange and reorientation speed of molecular movements, it can be used to detect cross-linking in polymers [38, 39]. Cross-linking produces a more rigid structure with anisotropic chain motions, which gives broadened line widths in NMR spectra. For example, O’Donnell and Whittaker [40] have studied cross-linking in cis-polybutadiene and shown that the line widths increased significantly upon $\gamma$-irradiation (or with increasing crosslink density); Yu et al. [41] observed a drastically increased line width in HP\&HT cross-linked (vinyl) 1,2-polybutadiene using solid-state ${ }^{13} \mathrm{C}$ CP NMR. However, in this case, the line widths of the F-composite's spectrum did not increase after the $1.7 \mathrm{GPa}$ treatment, which indicates that nylon-6 does not become cross-linked. This corroborates previous results of lack of cross-links in pure nylon-6 samples after treatment for $4 \mathrm{~h}$ at $530 \mathrm{~K}$ at 1.0 and $1.2 \mathrm{GPa}$, which was deduced from the absence of gel formation after dissolving in formic acid [8]. Thus, the higher treatment pressure and inclusion of lightly functionalized CNTs did not affect this result.

\subsection{Degree of crystallinity, thermal stability and structural properties by DSC}

DSC was applied to establish the melting temperature $\left(T_{\text {melt }}\right)$, transition enthalpy $(\Delta \mathrm{H})$, crystallization temperature $\left(T_{\text {cry1 }}, T_{\text {cry2 }}\right)$ before and after HP\&HT treatment. As shown in Fig. 4, both virgin and HP\&HT treated samples exhibited an endothermic melting peak at ca. $220{ }^{\circ} \mathrm{C}$ on heating, followed by an exothermic crystallization dip at ca. $190{ }^{\circ} \mathrm{C}$ on cooling. The data from the first and second heating and cooling runs are listed in Tables 2 and 3.

On the first heating of the untreated samples, nylon- 6 showed $T_{\text {melt }}$ of $222.6{ }^{\circ} \mathrm{C}$, the Pcomposite $T_{\text {melt }}=223.2{ }^{\circ} \mathrm{C}$ and the F-composite $T_{\text {melt }}=221.1{ }^{\circ} \mathrm{C}$. Since the samples had identical thermal histories [42], the differences must either be due to different properties of the nylon-6 matrix, e.g. different molecular weight, or an effect caused by the CNTs or a combination of these. The slightly higher $T_{\text {melt }}$ of the P-composite goes along with its larger $C S P$, whereas the lower value for the F-composite may be linked to the low CSP of $\alpha 2$. In any case, the MWCNT fillers do not strongly affect the melting temperature in untreated nylon-6. 


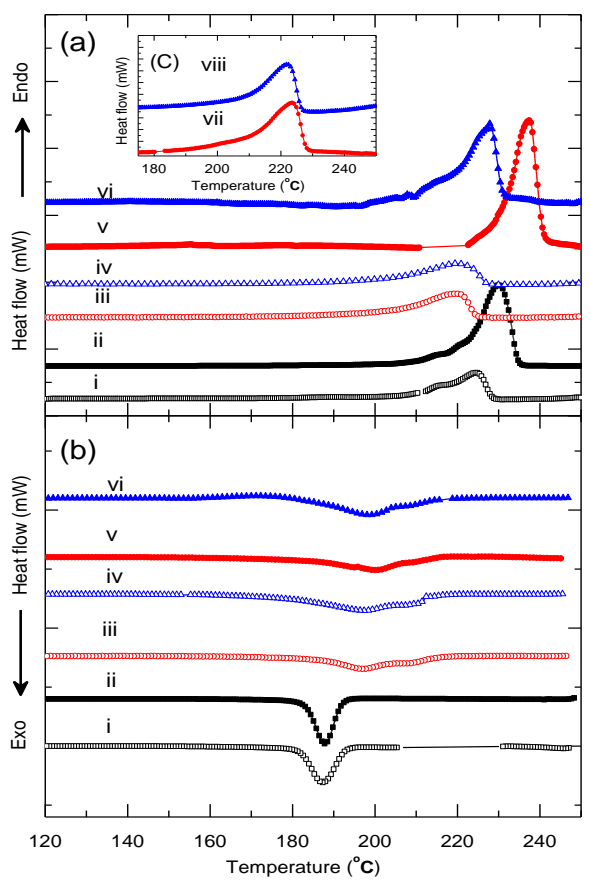

Fig. 4. DSC traces of nylon-6 and composites: (a) first heating runs, (from bottom to top) i. nylon-6; ii. 1.0 GPa treated nylon-6; iii. P-composite; iv. F-composite; v. 1.0 GPa treated Pcomposite; vi. 1.7 GPa treated F-composite. (b) First cooling runs, the sequence and label of spectra are the same as in (a). (c) Second heating runs of HP\&HT treated samples, vii. 1.0 GPa treated P-composite viii. 1.7 GPa treated F-composite. Scans are shifted vertically for clarity.

Table 2. DSC Results of the first heating and cooling runs. The values are averages for at least 2 samples except for the treated F-composite.

\begin{tabular}{lllll}
\hline Sample & $\Delta H(\mathrm{~J} / \mathrm{g})$ & $T_{\text {melt }}\left({ }^{\circ} \mathrm{C}\right)$ & $T_{\text {cry2 }}\left({ }^{\circ} \mathrm{C}\right)$ & $T_{\text {cry1 }}\left({ }^{\circ} \mathrm{C}\right)$ \\
\hline Nylon-6 & 50.6 & 222.6 & - & 185.5 \\
Treated nylon-6 & 136.9 & 226.9 & - & 187.7 \\
P-composite & 47.4 & 223.2 & 212.6 & 199.6 \\
Treated P-composite & 157.8 & 234.6 & 208.9 & 198.8 \\
F-composite & 68.6 & 221.1 & 209.1 & 195.8 \\
Treated F-composite & 105.8 & 224.8 & 208.0 & 197.0 \\
\hline
\end{tabular}


On the first cooling, nylon-6 exhibited an exothermic $T_{\text {cry1 }}$ dip at $185.5{ }^{\circ} \mathrm{C}$, whereas $T_{\text {cry } 1}$ of the P- and F-composites were significantly higher, 199.6 and $195.8^{\circ} \mathrm{C}$, respectively. In addition to these main crystallization exotherms, the DSC traces of the composites show weak exotherms at $\sim 10{ }^{\circ} \mathrm{C}$ above $T_{\text {cry1 }}$ (Fig. 4b), which we denote by $T_{\text {cry2 }}$. Moreover, the DSC results of the P- and F- composites show wide crystallization temperature ranges in sharp contrast to the narrow temperature range for neat nylon-6. The raise in the crystallization temperatures suggests that the MWCNTs act as nucleation sites and promote heterogeneous nucleation and lamellae growth near the CNT surface, as observed previously in other CNT/polymer systems [43, 44]. The much broader crystallization range for the composites can be due to the significantly higher crystallization temperature, which reduces the growth rate in nucleation-controlled crystallization [45], perhaps in combination with CNT obstruction of chain motions required for growth of nylon-6 crystals. Li et al. [46] used non-isothermal crystallization data and deduced that MWCNTs acted as effective nucleation agents but hindered diffusion and alignment of the nylon6 chains, which decreased the crystallization rate. Miri et al.’s [30] study of isothermal crystallization in MMT/nylon-6 composites at $203{ }^{\circ} \mathrm{C}$ showed that the crystallization rate decreased with increasing MMT content, which indicates that nanofillers can reduce the crystallization rate of nylon-6. On the other hand, Chen and Wu's [47] study of the isothermal crystallization rate in the $195-205^{\circ} \mathrm{C}$ range showed that nylon- 6 with carboxylic acid functionalized MWCNTs crystallized faster than neat nylon-6, at least up to $3 \mathrm{wt} \%$ fillers. The kinetics of the crystallization process of nylon- 6 depends on both the conditions and thermomechanical history [25], and fillers may accelerate or reduce the crystallization rate dependent on the inherent crystallization properties of nylon-6 as well as the filler properties. In a comparison between MWCNT filled nylon-6 and neat nylon-6 under identical conditions, MWCNTs may thus increase the crystallization rate at low degrees of supercooling, when the self-nucleation rate of nylon-6 is low, but decreases the rate at high degrees of supercooling. Strictly this should mean that, depended on the thermal treatment, the degree of crystallinity can both be improved and reduced by MWCNT fillers.

The splitting into two exothermic events ( $T_{\text {cry1 }}$ and $\left.T_{\text {cry2 }}\right)$ suggests two distinct crystallization processes. As described above, nylon- 6 exhibits two phases, $\gamma$ and $\alpha$, but the peaks are unlikely due to $\gamma$ and $\alpha$ crystallization, respectively. As clearly shown by the WAXD and NMR spectra, the P- and F-composites have predominantly $\alpha$ crystalline structure both before and after the 
HP\&HT treatment, and only the amount of the amorphous fraction differ. Moreover, crystallization into the more stable $\alpha$ phase occurs at higher temperatures than that into the $\gamma$ phase, at least for neat nylon-6. This seems to preclude that the two peaks are associated with first $\gamma$ crystallization and then $\alpha$ crystallization, which is the room temperature phase, as established by the NMR and WAXD analyses.

The crystallization behavior in MWCNT/nylon-6 composites have been investigated before [46-49], and two exothermic features have been observed in some studies [48, 49] but not in all $[46,47]$. The origin of these is not firmly established, but Phang et al. [49] noted that the high temperature peak was associated with the presence of MWCNTs and tentatively suggested that the two peaks were due to a two step crystallization process. Brosse et al. [48] provided a similar explanation and attributed the two peaks to: (i) crystallization in the fraction of the nylon-6 matrix, which was not in contact with the CNTs $\left(T_{\text {cry1 }}\right)$ and (ii) crystallization at the CNT surfaces, or trans-crystallization $\left(T_{\text {cry2 }}\right)$. They suggested that the latter causes the high temperature exotherm when trans-crystalline lamellae nucleate and grow perpendicular to the CNT surfaces with the chains parallel to the CNT axis. The lack of such feature above the main crystallization peak was attributed to poor dispersion. More specifically, Brosse et al. [48] found that addition of MWCNTs in the matrix (0.1-10 wt\%) caused both $T_{\text {cry1 }}$ and $T_{\text {cry2 }}$ to shift to higher temperatures. Concurrently, the $T_{\text {cry2 }}$ peak became more distinct whereas the $T_{\text {cry1 }}$ peak became weaker suggesting that MWCNTs promote the nucleation and growth of lamellae near the CNT surface (trans-crystalline lamellae).

After the HP\&HT treatment, the $T_{\text {cry2 }}$ feature became less distinct. We find two possible reasons for this behavior; MWCNTs agglomeration and remaining nylon-6 nuclei. Since the sample was not melted during the HP\&HT treatment, agglomeration seems less likely. Moreover, although $T_{\text {cry1 }}$ of our synthesized nylon-6 did not change significantly, we have previously found that $T_{\text {cry1 }}$ of a commercial nylon-6 sample increased more than $10{ }^{\circ} \mathrm{C}$ after HP\&HT treatment [8]. It is known that crystalline structures can remain and serve as nucleation centers despite pretreatment above the nominal melting temperature before recording of DSC cooling traces [50]. This is the most likely explanation for both the slight increase of $T_{\text {cry1 }}$ of neat nylon- 6 and the less distinct exothermic peak associated with the MWCNT induced nucleation and growth.

However, even though the MWCNTs provide nucleation sites in nylon-6, and therefore decrease supercooling, this does not necessarily promote the degree of crystallinity. In fact, the 
crystallinity can even decrease, e.g., due to the decreased crystallization rate. As shown in Table $1, C_{\mathrm{DSC}}, C_{\mathrm{WAXD}}$ and $C_{\rho}$ of the untreated P- and F-composites are almost the same as that of nylon6, which indicate that the MWCNTs do not strongly affect the crystallinity. The result should depend on thermal treatment, which has also been discussed in detail by Li et al [51]. They found higher crystallinity in MWCNT filled nylon-6 than in neat nylon-6 after cooling from melt at rates higher than $2-3 \mathrm{~K} / \mathrm{min}$, but the reverse result at lower rates.

After HP\&HT treatment, the thermal stability of the samples improved as shown by the increased $T_{\text {melt }}$ On the first heating run, the P-composite, F-composite and nylon-6 showed, respectively, $\sim 11, \sim 4$ and $\sim 4 \mathrm{~K}$ higher $T_{\text {melt }}$ than the corresponding untreated samples. In addition, the HP\&HT treated samples exhibited larger $\Delta \mathrm{H}$ and therefore also higher $C_{\mathrm{DSC}}$. The treated Pcomposite, F-composite and nylon-6 had $\sim 3, \sim 2$ and $\sim 3$ times larger $\Delta \mathrm{H}$, respectively (Fig. $4 \mathrm{a}$ ).

The DSC results suggest that MWCNTs augment the improved thermal stability shown by nylon-6 after HP\&HT treatment, at least in the case of the P-composite, which showed $\sim 8 \mathrm{~K}$ higher $T_{\text {melt }}$ and distinctly higher $\Delta \mathrm{H}$ than similarly treated nylon-6. The WAXD results show that the $\alpha$ phase is produced both during crystallization at atmospheric pressure and during the cold crystallization process, which is induced by the HP\&HT treatment [8]. As depicted by Fig.4a, the DSC spectrum of the treated P-composite shows a narrow and distinct melting peak suggesting that the nylon-6 in the composite formed enlarged lamellae, which are reinforced by the MWCNTs. Since the MWCNTs acts as sites for lamellae growth $[43,44,48]$ and the HP\&HT treatment apparently promotes the growth of larger crystals, the probability of lamellae bridges between the MWCNTs increases. Apparently the MWCNTs support the nylon- 6 crystalline structure, which provides thermal reinforcement that raises $T_{\text {melt }}$.

As shown in Table 1, all HP\&HT produced samples show an irreversible increase of density. The density increases due to the amorphous to crystalline conversion of nylon-6, but this effect seems insufficient to explain the more pronounced density increase for the composites. The results of $C_{D S C}$ and $C_{W A X D}$ taken together indicate about the same increase of crystallinity in the composites as in neat nylon-6, but still the irreversible densification of the composites is significantly larger. The calculated density increase of nylon-6 in the P-composite is $~ 4 \%$, whereas that of neat nylon-6 increased $2 \%$ which implies a MWCNT promoted increase of the nylon-6 density. It has been suggested that the interfacial contact between polymers and CNTs generally promotes wrapping and/or coating [52]. The composites studied here are produced 
under strongly densified conditions which may augment the MWCNT-nylon-6 interfacial interaction. Consequently, the wrapped and/or coated layer of nylon-6 chains may become more strongly attached to the MWCNTs, which augment the irreversible densification. A similar augmented densification was previously observed in SWCNT/polyisoprene composites [9] in which the HP\&HT treatment cause cross-linking instead of cold crystallization and therefore precludes that the effect is associated with a change in crystallinity.

On the second heating run (Table 3), the values for $T_{\text {melt }}$ of the P- and F-composites were only $\sim 1 \mathrm{~K}$ higher than that of the untreated states, and $\Delta \mathrm{H}$ decreased significantly compared to the values of the treated samples. This means that $T_{\text {melt }}$ became similar for all samples after the first heating and cooling runs, but that the crystallization temperature of the composites still differed from that of neat nylon-6 due to the nucleation effect of the MWCNTs.

Table 3. DSC results of the second heating and cooling runs. (The untreated samples showed the same values as the first heating and cooling runs to within $1 \mathrm{~K}$ )

\begin{tabular}{ccccc}
\hline Sample & $\Delta H(\mathrm{~J} / \mathrm{g})$ & $T_{\text {melt }}\left({ }^{\circ} \mathrm{C}\right)$ & $T_{\text {cry2 }}\left({ }^{\circ} \mathrm{C}\right)$ & $T_{\text {cry1 }}\left({ }^{\circ} \mathrm{C}\right)$ \\
\hline Treated P-composite & 74.0 & 223.9 & 210.6 & 199.2 \\
Treated F-composite & 51.1 & 222.5 & 209.5 & 197.5 \\
\hline
\end{tabular}

\section{Conclusions}

Multi-wall carbon nanotube/nylon-6 composites undergo an amorphous to $\alpha$ phase conversion process under high-pressure-high-temperature conditions. This transformation increased the crystallinity from about 31\% to 58\% after treatment for $4 \mathrm{~h}$ at $1.0 \mathrm{GPa}$ and $530 \mathrm{~K}$, which is about the same as for neat nylon-6. Moreover, this pressure induced cold crystallization caused an increase of crystal sizes and a preferred crystal orientation. The new state show higher melting point and the increase is augmented by the presence of MWCNTs. The improved thermal stability is attributed to the highly crystalline nylon-6 structure reinforced by inter-crystal bridges of MWCNTs. NMR spectra of the high-pressure treated composites resemble that of single crystal nylon-6, which corroborate the results of an improved degree of crystallinity and a preferred crystal orientation, but there is no NMR visible indication of new covalent bonds between either nylon-6 molecules or nylon-6 and the MWCNTs. 


\section{Acknowledgement}

We are grateful for financial support from Magn. Bergvalls foundation, Kempe foundation, the

Swedish Research Council, and Knuth and Alice Wallenberg foundation.

\section{References}

1. Prassides $\mathrm{K}$ and Sundqvist B. Polymeric Fullerene Phases Formed Under Pressure. Fullerene-Based Materials, vol. 109: Springer Berlin / Heidelberg, 2004. pp. 85-126.

2. Blank VD, Buga SG, Dubitsky GA, R. Serebryanaya N, Popov MY, and Sundqvist B. Carbon 1998;36(4):319-43.

3. Nagel P, Pasler V, Lebedkin S, Soldatov A, Meingast C, Sundqvist B, Persson PA, Tanaka T, Komatsu K, Buga S, and Inaba A. Phys. Rev. B 1999;60(24):16920.

4. Tonpheng B, Yu J, and Andersson O. Phys. Chem. Chem. Phys. 2011;13(33):15047-54.

5. Bellander M, Stenberg B, and Persson S. Polym. Eng. Sci. 1998;38(8):1254-60.

6. $\quad$ Tonpheng B and Andersson O. Eur. Polym. J. 2008;44(9):2865-73.

7. Kurtz SM. UHMWPE biomaterials handbook : ultra high molecular weight polyethylene in total joint replacement and medical devices, 2nd ed. Amsterdam: Elsevier, 2009.

8. $\quad$ Yu J, Tonpheng B, and Andersson O. Macromolecules 2010;43(24):10512-20.

9. Tonpheng $\mathrm{B}, \mathrm{Yu} \mathrm{J}$, Andersson BM, and Andersson $\mathrm{O}$. Macromolecules 2010;43(18):7680-8.

10. Zhang WD, Shen L, Phang IY, and Liu T. Macromolecules 2004;37(2):256-9.

11. Qu L, Veca LM, Lin Y, Kitaygorodskiy A, Chen B, McCall AM, Connell JW, and Sun YP. Macromolecules 2005;38(24):10328-31.

12. Khalid Saeed S-YP. J. Appl. Polym. Sci. 2007;106(6):3729-35.

13. Jose MV, Steinert BW, Thomas V, Dean DR, Abdalla MA, Price G, and Janowski GM. Polymer 2007;48(4):1096-104.

14. Haggenmueller R, Guthy C, Lukes JR, Fischer JE, and Winey KI. Macromolecules 2007;40(7):2417-21.

15. Ha H, Kim S, and Ha K. Macromol. Res. 2010;18(7):660-7.

16. Gao J, Zhao B, Itkis ME, Bekyarova E, Hu H, Kranak V, Yu A, and Haddon RC. J. Am. Chem. Soc. 2006;128(23):7492-6.

17. Gao J, Itkis ME, Yu A, Bekyarova E, Zhao B, and Haddon RC. J. Am. Chem. Soc. 2005;127(11):3847-54.

18. Sandler JKW, Pegel S, Cadek M, Gojny F, van Es M, Lohmar J, Blau WJ, Schulte K, Windle AH, and Shaffer MSP. Polymer 2004;45(6):2001-15.

19. Yu J, Tonpheng B, Gröbner G, and Andersson O. Carbon 2011;49(14):4858-66.

20. Moon J-M, An KH, Lee YH, Park YS, Bae DJ, and Park G-S. J. Phys. Chem. B 2001;105(24):5677-81.

21. The samples were weighted again after measurement to make sure there is no water absorbed.

22. Isasi JR, Mandelkern L, Galante MJ, and Alamo RG. J. Polym. Sci. Part B: Polym. Phys. 1999;37(4):323-34.

23. Lewis ELV and Ward IM. J. Macromol. Sci., Part B: Phys. 1980;18(1):1-46.

24. $\quad$ Lin L and Argon AS. Macromolecules 1992;25(15):4011-24. 
25. Kohan MI. Nylon Plastics Handbook. Cincinnati: Hanser/Gardner Publications, Inc. , 1995.

26. Gogolewski S and Pennings AJ. Polymer 1977;18(7):647-53.

27. Li X, Yuan G, Westwood A, Zhang H, Dong Z, Brown A, Brydson R, and Rand B. Chem. Vap. Deposition 2008;14(1-2):40-5.

28. Liu, Phang IY, Shen L, Chow SY, and Zhang W-D. Macromolecules 2004;37(19):721422.

29. Lincoln DM, Vaia RA, and Krishnamoorti R. Macromolecules 2004;37(12):4554-61.

30. Miri V, Elkoun S, Peurton F, Vanmansart C, Lefebvre JM, Krawczak P, and Seguela R. Macromolecules 2008;41(23):9234-44.

31. Lin S-Y, Chen E-C, Liu K-Y, and Wu T-M. Polym. Eng. Sci. 2009;49(12):2447-53.

32. Kubo K, Yamanobe T, Komoto T, Ando I, and Shiibashi T. J. Polym. Sci. Part B: Polym. Phys. 1989;27(4):929-37.

33. Hatfield GR, Glans JH, and Hammond WB. Macromolecules 1990;23(6):1654-8.

34. Kubo K, Ando I, Shiibashi T, Yamanobe T, and Komoto T. J. Polym. Sci. Part B: Polym. Phys. 1991;29(1):57-66.

35. VanderHart DL, Asano A, and Gilman JW. Chem. Mater. 2001;13(10):3781-95.

36. Yang J, Dong W, Luan Y, Liu J, Liu S, Guo X, Zhao X, and Su W. J. Appl. Polym. Sci. 2002;83(12):2522-7.

37. Yang J, Liu S, Guo X, Luan Y, Su W, and Liu J. Macromol. Chem. Phys. 2002;203(8):1081-7.

38. Mohanraj S and Ford WT. Macromolecules 1985;18(3):351-6.

39. Errede LA, Newmark RA, and Hill JR. Macromolecules 1986;19(3):651-4.

40. O'Donnell JH and Whittaker AK. J. Polym. Sci. Part A: Polym. Chem. 1992;30(2):18595.

41. Yu J, Tonpheng B, Grobner G, and Andersson O. J. Mater. Chem. 2011;21(35):13672-82.

42. The samples were first melted by heating to $250{ }^{\circ} \mathrm{C}$ at $10 \mathrm{~K} / \mathrm{min}$, annealed at $250{ }^{\circ} \mathrm{C}$ for 5 min and then cooled to $20^{\circ} \mathrm{C}$ at $10 \mathrm{~K} / \mathrm{min}$ rate before the DSC scan started.

43. Grady BP, Pompeo F, Shambaugh RL, and Resasco DE. J. Phys. Chem. B 2002;106(23):5852-8.

44. Valentini L, Biagiotti J, Kenny JM, and Santucci S. J. Appl. Polym. Sci. 2003;87(4):70813.

45. Mandelkern L. Crystallization of polymers:Kinetics and mechanisms, 2nd ed. Cambridge: University Press, 2004.

46. Li J, Fang Z, Tong L, Gu A, and Liu F. Eur. Polym. J. 2006;42(12):3230-5.

47. Chen E-C and Wu T-M. J. Polym. Sci. Part B: Polym. Phys. 2008;46(2):158-69.

48. Brosse A-C, Tencé-Girault S, Piccione PM, and Leibler L. Polymer 2008;49(21):4680-6.

49. In Yee Phang, Ma J, Shen L, Liu T, and Zhang W-D. Polym. Int. 2006;55(1):71-9.

50. Aharoni SM. n-Nylons: their synthesis, structure, and properties. Chichester: John Wiley \& Sons, Ltd., 1997.

51. Li J, Fang Z, Tong L, Gu A, and Liu F. J. Polym. Sci. Part B: Polym. Phys. 2006;44(10):1499-512.

52. Baskaran D, Mays JW, and Bratcher MS. Chem. Mater. 2005;17(13):3389-97. 